\title{
Energetics of cobalt alloys and compounds and solute-vacancy binding in fcc cobalt: A first-principles database
}

\author{
S. Shahab Naghavi, Vinay I. Hegde, Abhinav Saboo, C. Wolverton* \\ Department of Materials Science and Engineering, Northwestern University, Evanston, \\ Illinois 60208, USA
}

\begin{abstract}
Using extensive first-principles density functional calculations, we calculate thermodynamic properties of binary fcc cobalt-based alloys with 25 different solute elements. For each solute element $X$, we calculate its (a) nearest- and nextnearest-neighbor solute-vacancy binding energy, (b) dilute impurity mixing energy with respect to the equilibrium and hypothetical fcc-based reference states of the solute, (c) enthalpy of formation of cobalt-rich binary ordered compounds in the Co- $X$ system, (d) solubility enthalpy, and other derived quantities. We find that the solute-vacancy binding energies of all the studied solutes in fcc cobalt are positive (indicating favorable binding), in contrast to nickel, aluminum, magnesium, and copper-based alloys, where mid $3 d$ transition metal solutes have unfavorable solute-vacancy binding. We study the physical and chemical effects influencing solute-vacancy binding energy, and find that (a) it correlates broadly with solute size - larger solute atoms possess stronger binding with vacancies - and this is understood in terms of strain relief and secondary next-nearest-neighbor interactions, and (b) it follows a parabolic trend as a function of $d$-occupancy across the transition metal series - falling to a minimum at the middle of the series - and this is understood in terms of a $d$-band filling effect. We also find this $d$-band filling effect in other calculated quantities
\end{abstract}

\footnotetext{
*Corresponding Author

Email address: c-wolverton@northwestern.edu (C. Wolverton)
}

Preprint submitted to Acta Materialia

October 24, 2016

(C) 2016. This manuscript version is made available under the Elsevier user license http://www.elsevier.com/open-access/userlicense/1.0/ 
such as the dilute impurity volumes and dilute impurity mixing energies. To aid in building thermodynamic databases, we tabulate all the calculated thermodynamic quantities, and compare them with experimental phase diagrams data and previous literature, and find good agreement where such data is available.

\section{Introduction}

The field of superalloys (high-performance alloys) has received a spike in interest following the discovery of $\gamma^{\prime}-\mathrm{Co}_{3}(\mathrm{Al}, \mathrm{W})$ precipitates with the $\mathrm{L}_{2}$ structure [1], promising a new class of Co-based high-temperature materials. These 5 novel superalloys offer better oxidation, corrosion, and wear resistance and potentially higher operation temperatures compared to Ni-based superalloys $[2$, 3]. Despite having some superior chemical and physical properties [1, 4], alloys based on $\mathrm{Co}_{3}(\mathrm{Al}, \mathrm{W})$ suffer from several problems such as inferior hightemperature strength $[5,6]$, a narrow $\gamma+\gamma^{\prime}$ two-phase region, and a low stacking fault energy [6]. Alloying additions are an effective way, at least in part, to overcome these problems and improve the mechanical properties of Co-based superalloys [3, 5-10]. For example, the addition of Ta, Ti, Nb, Hf, or Ni [3, 9-12] increases the stability and the stacking fault energy of $\gamma^{\prime}-\mathrm{Co}_{3}(\mathrm{Al}, \mathrm{W})$ simultaneously, thus improving the high-temperature strength of the $\gamma^{\prime}$ phase and $\gamma+\gamma^{\prime}$ two-phase superalloys [12].

To understand the effects of alloying additions on diffusion in fcc cobalt, a detailed knowledge of the interaction between a solute atom and an accompanying vacancy (hereafter, a vacancy is indicated by $\square$ ) is necessary [13-15]. The solute $-\square$ binding energy directly influences impurity diffusion. In an alloy with dilute solute concentrations, the solute $-\square$ binding energy controls the probability of having a vacancy at a lattice site neighboring a solute atom. Therefore, in a vacancy-mediated diffusion process, energetic binding between a solute atom and a vacancy is a key factor in the quantitative understanding of solute diffusion [13] and as well as other associated kinetic phenomena such as precipitate nucleation. For example, the observed decreased response to natural aging with 
microalloying additions (e.g., the behavior of $\mathrm{Al}-\mathrm{Cu}$ alloys with the microalloying addition of Sn) could be understood in terms of vacancy trapping, due to a strong solute- $\square$ binding energy $[13,14]$. Despite its importance, this interaction is notoriously difficult to experimentally measure accurately [16] but can be readily calculated using first-principles electronic structure methods such as density functional theory (DFT) $[14,15,17,18]$. To our knowledge no such calculations exist for Co-based alloys.

In addition to kinetic phenomena, the thermodynamics of alloying behavior is also critically important in the design of new Co-based alloys. Among the computational tools available today for studying thermodynamic phase stability in alloys, calculation of phase diagrams (CALPHAD) methods are one of the most widely used [19-21]. These methods use databases of thermodynamic functions, and can accurately calculate phase diagrams of multicomponent alloys. While CALPHAD methods have been successfully applied over the years 40 to many commercial alloy systems and databases (e.g., Al-based alloys, Fe-based alloys), mature databases for similar efforts in developing Co-based superalloys are not currently available [22]. In cases where experimental data is unavailable, DFT calculations can provide a wealth of energetic and thermodynamic information (e.g., mixing energies of solid solutions, formation energies of ordered 45 compounds, etc.) $[23,24]$ that significantly complement CALPHAD approaches. Thus, an extensive first-principles investigation of thermodynamics in cobalt alloys would greatly accelerate the development of CALPHAD databases, and ultimately, the design of new Co-based superalloys.

In the present work, the solute- $\square$ binding energy, dilute mixing energy, dilute volume of mixing, compound formation energy, and solubility enthalpy of 25 substitutional solutes in fcc cobalt are calculated using DFT. We include all of the alloying elements used in Co-based superalloys [6] which consist of almost all $3 d, 4 d$, and $5 d$ transition metal elements - except groups 3 and 12 - together with silicon and aluminum. Some of the alloying elements considered, such as technetium and silver, may have no practical interest but provide useful data that serves to find systematic trends across the periodic table. We analyze the 
results of our calculations to search for the key physical effects that influence solute $-\square$ binding, such as $d$-occupancy, solute size, and second nearest-neighbor interactions. Finally, in an effort to aid in building thermodynamic databases, we tabulate all the calculated thermodynamic properties and compare them with experimental phase diagrams data, where available.

\section{Methodology}

\subsection{Density Functional Theory}

For calculations in the present work, we used plane-wave DFT with projectoraugmented plane-wave (PAW) method [25, 26] as implemented in Vienna Abinitio Simulation Package (VASP) [27, 28]. The exchange-correlation energy functional was described with the spin-polarized generalized gradient approximation of Perdew-Burke-Ernzerhof (PBE) [29]. We used the PAW potentials recommended by VASP, with a plane wave cutoff energy 1.3 times the maximum energy cutoff ("ENMAX") listed in the PAW potentials for each system. We verified the convergence of the calculated quantities with respect to the plane wave cutoff energy by calculating all energies at a constant large cutoff of $520 \mathrm{eV}-$ and found that the change in the solute $-\square$ binding energies was negligible ( $<$ $0.2 \%)$. We relaxed all structures fully, with respect to cell lattice vectors and 75 atomic positions, by minimizing the energy until the Hellmann-Feynman forces on all atoms were less than $0.01 \mathrm{eV} / \AA$, and stresses on the cell were a few kbar or less. We then used this relaxed structure for a final "static" self-consistent run to calculate the total energy. Therefore, all calculations were relaxed under constant zero pressure.

We utilized a periodic supercell in which one host atom is replaced with either a solute or a vacancy to approximate dilute limits. As discussed in Ref. [15], a large size mismatch between the solute/defect and the host atoms can result in elastic strain on nearby atoms, in addition to spurious defect-defect interaction due to periodic boundary conditions, and minimizing this interaction requires very large supercells. Here, we found that a 108-atom supercell consisting of 
$3 \times 3 \times 3$ cubic conventional cells, which separates impurity atoms by $\approx 11 \AA$, results in solute- $\square$ binding energies converged to within $1 \mathrm{meV} /$ solute atom. We performed integrations over the Brillouin zone of the 108-atom supercell using the first-order Methfessel-Paxton [30] approach with a smearing width of $0.2 \mathrm{eV}$

90 and a $3 \times 3 \times 3$ Monkhorst-Pack [31] k-mesh during structural relaxation, and the tetrahedron method with Blöchl [32] corrections and a $4 \times 4 \times 4$ Monkhorst-Pack $\mathrm{k}$-mesh during the final static calculation for accurate total energies.

\subsection{Calculation of Energetic Quantities}

We calculate the energetics of solute $-\square(X-\square)$ binding for various pair spacings (e.g., nearest and second-nearest neighbors). The binding energy $E_{\text {bind }}$ is defined as the difference between the energy of "infinite separation" and nearestneighbor (or second-nearest-neighbor) separation of the solute and the vacancy. We calculated this difference using four independent calculations as follows:

$$
\begin{aligned}
-E_{\mathrm{bind}}(X-\square)= & \left(E\left(\mathrm{Co}_{N}\right)-E\left(\mathrm{Co}_{N-1} \square_{1}\right)\right) \\
& -\left(E\left(\mathrm{Co}_{N-1} X_{1}\right)-E\left(\mathrm{Co}_{N-2} X_{1} \square_{1}\right)\right)
\end{aligned}
$$

where $X$ and $\square$ represent a solute and vacancy respectively, embedded in an ${ }_{95} N=108$-atom supercell. The two groups of terms on the right side of Eq. 1 represent the energy required to break a host-host bond (first term) and a hostimpurity bond (second term) due to the introduction of a vacancy. Thus, the solute- $\square$ binding energy can be qualitatively understood as the difference between the host-impurity and host-host bond energies. In other words, if the host-impurity $(\mathrm{Co}-X)$ interaction is weaker than the host-host $(\mathrm{Co}-\mathrm{Co})$ interaction, a favorable solute $-\square$ binding can be expected. The negative sign of $E_{\text {bind }}$ in Eq. 1 is included to follow standard convention: a positive value corresponds to a favorable binding between the solute and the vacancy.

By having all the four energy terms on the right side of Eq. 1, one can easily calculate as a "by-product", the vacancy formation energy in fcc cobalt:

$$
E_{\mathrm{vac}}(\square)=E\left(\mathrm{Co}_{N-1} \square_{1}\right)-\frac{N-1}{N} E\left(\mathrm{Co}_{N}\right)
$$


Similarly, the dilute impurity mixing energy of a solute $X$ in fcc cobalt is also readily obtained using:

$$
E_{\mathrm{imp}}(X)=E\left(\mathrm{Co}_{N-1} X_{1}\right)-\frac{N-1}{N} E\left(\mathrm{Co}_{N}\right)-E(X)
$$

where $E_{\text {imp }}(X)$ is calculated for both $X$ in the fcc $\left(E^{\mathrm{fcc}}(X)\right)$ and its equilibrium crystal structure $\left(E^{\mathrm{eq}}(X)\right)$.

In order to get a quantitative measure of the atomic size of each solute impurity, $X$, we also calculate the dilute volume of mixing (hereafter, simply "impurity volume"), $V_{\text {imp }}^{X}$, which is given by the difference in volume induced by substituting a single fcc cobalt atom with a solute atom:

$$
V_{\mathrm{imp}}^{X}=V\left(\mathrm{Co}_{N-1} X_{1}\right)-V\left(\mathrm{Co}_{N}\right)
$$

We note here that after adding a solute atom to the fcc cobalt matrix, we fully relaxed the alloyed structure to its ground state; therefore, this definition of the impurity volume includes all chemical interactions between a solute atom and the nearby cobalt atoms and thus is different from the standard atomic volume of $X$.

The equilibrium formation enthalpy of a binary ordered compound is the energy of the compound relative to the pure elements, each in their equilibrium crystal structure:

$$
\Delta H_{\mathrm{ord}}^{\mathrm{eq}}\left(\mathrm{Co}_{p} X_{q}\right)=E\left(\mathrm{Co}_{p} X_{q}\right)-\left\{(1-x) E^{\mathrm{eq}}(\mathrm{Co})+x E^{\mathrm{eq}}(X)\right\}
$$

where $E\left(\mathrm{Co}_{p} X_{q}\right)$ is the ground state energy (per atom) of the ordered $\mathrm{Co}_{p} X_{q}$ compound and $x$ is the atomic fraction of $X, \frac{q}{p+q}$, in the compound.

Finally, the solubility enthalpy of a solute $X$ is given by the difference (per solute atom) between the equilibrium formation enthalpy of the Co-rich binary ordered compound (from Eq. 5) and the dilute impurity mixing energy (from Eq. 3):

$$
\Delta H_{\mathrm{sol}}(X)=\Delta H_{\mathrm{ord}}^{\mathrm{eq}}\left(\mathrm{Co}_{p} X_{q}\right)-E_{\mathrm{imp}}(X)
$$

Using $\Delta H_{\text {sol }}$, one can calculate the dilute solubility of a solute $X\left(x_{0}(\mathrm{X})\right)$ at a 
given temperature $T$ :

$$
x_{0}(X)=\exp \left(\Delta H_{\mathrm{sol}} / k_{\mathrm{B}} T\right) \cdot \exp \left(-\Delta S_{\mathrm{sol}} / k_{\mathrm{B}}\right)
$$

where $\Delta S_{\text {sol }}$ is the difference in the non-configurational entropy (e.g., vibrational, magnetic, etc.) between the ordered and disordered states (i.e., the non-configurational entropy difference analogous to Eq. 6). The entropic term $\exp \left(-\Delta S_{\mathrm{sol}} / k_{\mathrm{B}}\right)$ can act as an enhancement factor [33] in solubility calculations. Previous studies [33-35] on the solubility of $\mathrm{Sc}, \mathrm{Cu}$, and rare-earth elements in aluminum have demonstrated that an excellent agreement between calculated and experimental solubility can be achieved by calculating and adding vibrational entropy to Eq. 7. Nevertheless, the solubility enthalpy alone can help distinguish between solutes with high and low solubility [36]. We defer the calculation of the nonconfigurational entropy to future work.

\section{Results and Discussion}

\subsection{Solute-Vacancy Binding Energies}

To study interactions between a solute and a vacancy in detail, both the nearest-neighbor (NN) and the next-nearest-neighbor (2NN) solute- $\square$ binding energies were calculated. Table 1 lists NN, 2NN solute- $\square$ binding energies for all the 25 solute elements studied (consisting of 23 transition metal and 2 maingroup elements). As transition metal solutes are the main focus of this work, we analyze their energetics in great detail, and only briefly comment on the solute- $\square$ binding of the 2 main-group elements, namely aluminum and silicon. Overall, in contrast to $\mathrm{Ni}, \mathrm{Al}, \mathrm{Mg}$, and $\mathrm{Cu}$ alloys in which solute- $\square$ binding energies of mid $3 d$ transition metal solutes are negative or nearly zero [14, 17, 18, 37], we calculate all of them to be positive in fcc Co, i.e., indicating favorable binding. We also note that Neumerier et al. [38] recently reported $\mathrm{NN}$ and $2 \mathrm{NN}$ solute- $\square$ binding energies for Re, Mo, Ta, and $\mathrm{W}$ in fcc cobalt - the corresponding binding energies from our calculations are in excellent agreement (within $0.01 \mathrm{eV}$ ). We discuss the physical and chemical factors that govern the solute- $\square$ binding energies, namely, solute size and $d$-occupancy. We focus mainly on the NN 

solute- $\square$ energies and beyond.

\subsubsection{Effect of Solute Size}

Main-Group Solutes. The solute- $\square$ binding energies of aluminum and silicon in fcc cobalt are listed in Table 1 . The calculated binding of the solute atom

to an accompanying vacancy is positive, and about $0.1 \mathrm{eV}$, for both $\mathrm{Al}$ and Si. As discussed in Sec. 2.2, such a favorable binding indicates that the $s p-d$ bond formed between the solute and the host is weaker than the $d-d$ bonds between host atoms, and thus creating a vacancy next to a solute atom is easier than creating one next to a cobalt atom. But, as seen in Fig. 1, a direct correlation between the (main-group) solute size and its energetic binding to a vacancy is not apparent- $\mathrm{Al}$ is bigger than $\mathrm{Co}$, while $\mathrm{Si}$ is smaller, but they both have similar positive binding energies. As the chemistry of $s p-d$ bonds is different from that of $d-d$ ones [39, 40], a direct comparison of the change in volume due to main-group and transition metal solutes cannot be made ei5 previously observed $[14,15,17]$ that transition metal solutes do not show a systematic trend in solute- $\square$ binding energies with solute size; and vice versa, in transition metal hosts such as $\mathrm{Cu}$, binding energies of main-group solutes do not vary systematically with impurity volume [18]. Since we only consider two main-group elements as solutes, and a detailed analysis of the physics of the $s p-d$ interactions is beyond the scope of this work, we will limit the rest of our discussion to transition metal solutes.

Transition Metal Solutes. Fig. 1 shows the calculated solute- $\square$ binding energies as a function of the solute volume. We find a fair correlation between the binding energy of a solute and its size. Here we analyze this correlation for all the transition metal solutes.

To simplify our analysis, we consider a two-dimensional (2D) subspace of a (100) plane of fcc cobalt, containing a vacancy and a solute atom. As seen in 
Table 1: List of elemental solutes considered in this work, their respective dilute impurity volumes $\left(V_{\mathrm{imp}}\right)$, and $\mathrm{NN}$ and $2 \mathrm{NN}$ solute- $\square$ binding energies.

\begin{tabular}{|c|c|c|c|c|}
\hline & Solute & $\begin{array}{c}V_{\text {imp }} \\
\left(\AA^{3} / \text { solute }\right)\end{array}$ & NN & $\begin{array}{l}2 \mathrm{NN} \\
\mathrm{V})\end{array}$ \\
\hline & $\mathrm{Al}$ & 1.78 & 0.10 & -0.04 \\
\hline & $\mathrm{Si}$ & -1.11 & 0.13 & -0.00 \\
\hline \multirow{7}{*}{$\vec{D}$} & $\mathrm{Ti}$ & 3.15 & 0.16 & -0.09 \\
\hline & $\mathrm{V}$ & 0.75 & 0.04 & -0.03 \\
\hline & $\mathrm{Cr}$ & -0.23 & 0.01 & -0.03 \\
\hline & $\mathrm{Mn}$ & 0.23 & 0.01 & -0.05 \\
\hline & $\mathrm{Fe}$ & 0.67 & 0.02 & -0.03 \\
\hline & $\mathrm{Ni}$ & 0.52 & 0.02 & 0.00 \\
\hline & $\mathrm{Cu}$ & 1.44 & 0.09 & -0.02 \\
\hline \multirow{8}{*}{ 감 } & $\mathrm{Zr}$ & 9.28 & 0.46 & -0.16 \\
\hline & $\mathrm{Nb}$ & 4.88 & 0.24 & -0.09 \\
\hline & Mo & 3.39 & 0.10 & -0.04 \\
\hline & $\mathrm{Tc}$ & 4.23 & 0.03 & -0.00 \\
\hline & $\mathrm{Ru}$ & 4.76 & 0.05 & 0.01 \\
\hline & $\mathrm{Rh}$ & 4.06 & 0.08 & -0.00 \\
\hline & $\mathrm{Pd}$ & 4.63 & 0.15 & -0.04 \\
\hline & $\mathrm{Ag}$ & 5.89 & 0.28 & -0.07 \\
\hline \multirow{8}{*}{ Dי } & Hf & 8.07 & 0.38 & -0.15 \\
\hline & $\mathrm{Ta}$ & 5.40 & 0.19 & -0.09 \\
\hline & $\mathrm{W}$ & 3.41 & 0.06 & -0.05 \\
\hline & $\operatorname{Re}$ & 3.77 & 0.01 & 0.01 \\
\hline & Os & 4.95 & 0.02 & 0.04 \\
\hline & Ir & 4.90 & 0.07 & 0.03 \\
\hline & $\mathrm{Pt}$ & 5.32 & 0.13 & 0.00 \\
\hline & $\mathrm{Au}$ & 6.76 & 0.27 & -0.04 \\
\hline
\end{tabular}




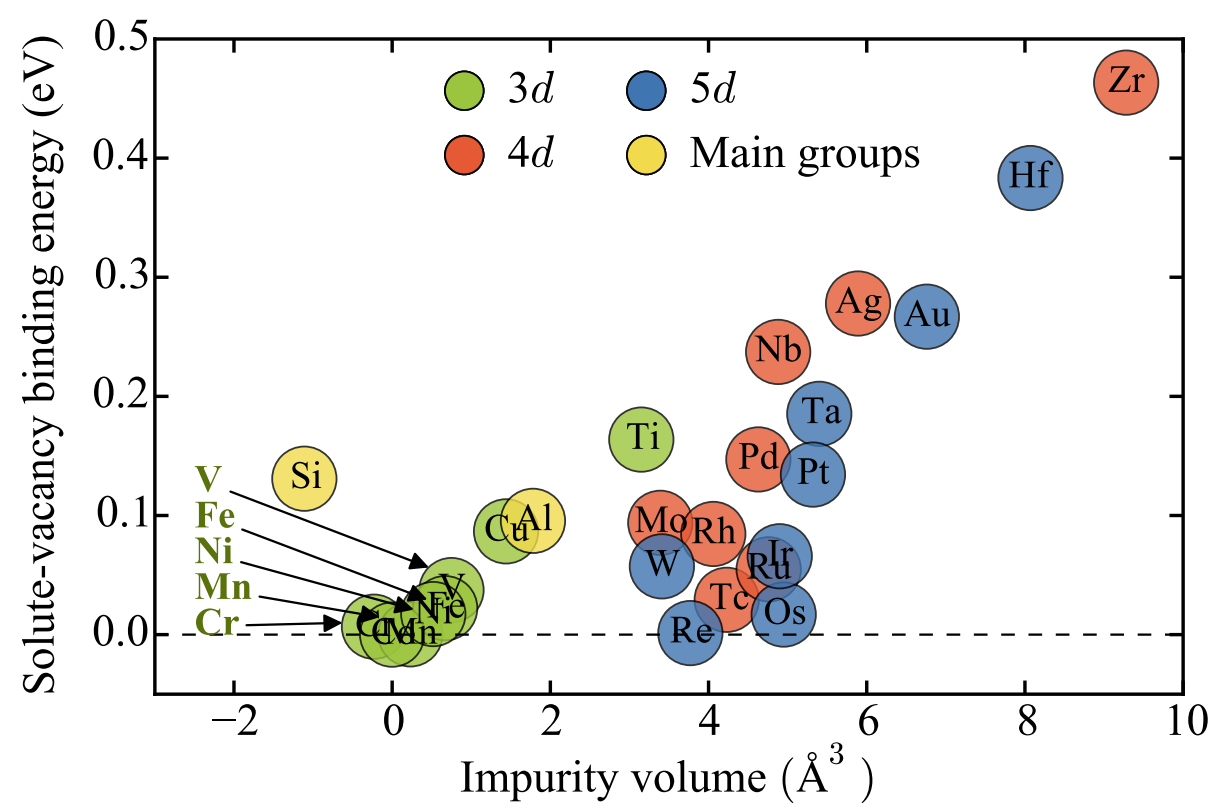

Figure 1: (Color online) The calculated NN solute- $\square$ binding energies as a function of impurity volume for all the solutes considered in this work. There is a modest correlation between solute- $\square$ binding and the size of the impurity: large solutes bind to vacancies more strongly than small ones, in general. 


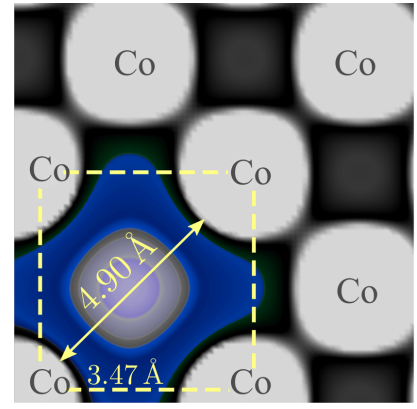

(a)

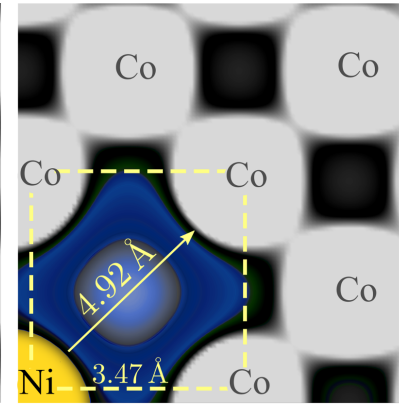

(b)

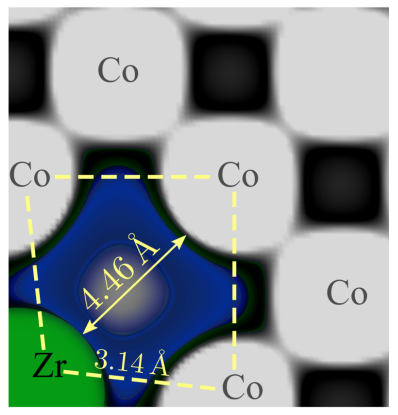

(c)

Figure 2: (Color online) A contour plot of the charge density on a (100) plane of $\mathrm{Co}_{106} \mathrm{X}_{1} \square_{1}$, where the solute $X$ is (a) a Co atom itself (i.e., no solute), (b) a solute atom of size similar to that of $\mathrm{Co}$, such as $\mathrm{Ni}$, and (c) a large solute atom, such as Zr. The dark blue region represents the void due to a vacancy. The length of this void (i.e., Co-Co distance passing directly through the vacant site) in $\mathrm{Co}_{107} \square_{1}$ is about $4.90 \AA$. Large solute atoms such as Zr relax toward the vacancy by up to $\approx 0.45 \AA$, significantly deforming the geometry of the structure, while solute atoms of the same size as $\mathrm{Co}$, such as $\mathrm{Ni}$, keep the local geometry virtually intact.

Fig. 2, in this (100) plane, we can study the interaction of a vacancy with four of its nearest-neighbor atoms. In fact, one can easily generalize the following discussion to the three orthogonal planes that include all 12 nearest-neighbor atoms of the fcc lattice. The distance between the NN cobalt atoms is $2.48 \AA$ and twice this distance (the $4 \mathrm{NN}$ separation), the face-diagonal of the fcc cube, is $4.96 \AA$. The four atoms located at the corners of the $2 \mathrm{D}$ body-centered arrangement are $2 \mathrm{NN}$ of each other, separated by $3.51 \AA$. As seen in Fig. 2(a), once the central cobalt atom is removed, creating a vacancy $\left(\mathrm{Co}_{N-1} \square_{1}\right)$, the four $\mathrm{NN}$ atoms at the corners relax toward the center by $\approx 0.03 \AA$, reducing the $4 \mathrm{NN}$ distance from 4.96 to $4.90 \AA$ and the $2 \mathrm{NN}$ distances (the side of the square) from 3.51 to $3.47 \AA$. Here, we address how a solute atom affects the local environment of a NN vacancy.

A solute atom exerts a strain on all the NN and $2 \mathrm{NN}$ host atoms and the magnitude of this strain depends directly on the solute size and the binding between the solute and host atoms. To reduce this strain, the NN host atoms 


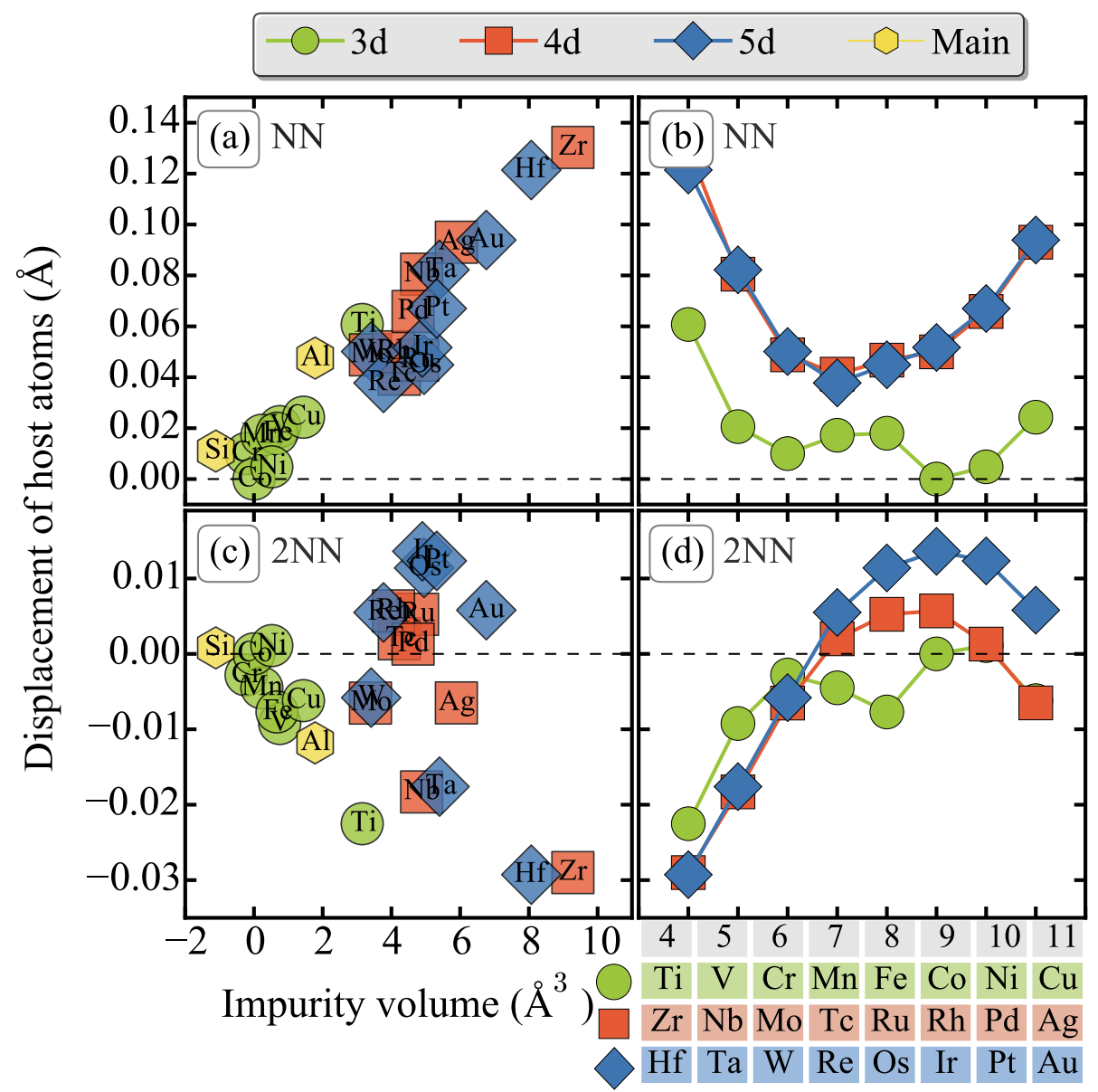

Figure 3: (Color online) The effect of volume and $d$-occupancy of a substitutional impurity on the atomic relaxation of the (a,b) 12 nearest-neighbor atoms (NN) and (c,d) 6 next-nearestneighbor atoms $(2 \mathrm{NN})$ atoms. The displacements are calculated with respect to fully-relaxed pure fcc cobalt, for which NN and 2NN distances are 2.48 and $3.51 \AA$ respectively. Therefore, here, negative and positive displacements correspond to attractive and repulsive interactions respectively. 
tend to relax away from the solute atom. Fig. 3 shows the displacement of the NN host atoms upon the introduction of a solute atom, relative to pure fcc cobalt. Solutes of size similar to Co, such as Ni, apply a negligible strain on the adjacent atoms resulting in displacements of only about $0.01 \AA$, which is in fact within the accuracy of DFT [41]. This small strain on the adjacent atoms due to the solute atom means that a vacancy next to it does not further reduce the strain; therefore, based only on strain, we would expect small solute- $\square$ binding energies for these solutes. In fact, almost all $3 d$ transition metals, with the exception of $\mathrm{Ti}$ and to some extent $\mathrm{Cu}$, belong to this category (with binding energies less than $\approx 0.05 \mathrm{eV}$ ). Not surprisingly, $\mathrm{Ti}$ and $\mathrm{Cu}$ show larger binding energies than the other $3 d$ transition metals (see Fig. 1).

Furthermore, substituting a large solute atom in fcc cobalt creates a large strain on the NN Co atoms, which relax away with displacements as large as $0.13 \AA$ for large solute atoms such as $\mathrm{Zr}$ (see Fig. 3). Here, creating a vacancy in the NN position allows the large solute atom to relax toward the vacancy, away from the other $11 \mathrm{NN}$ atoms, reducing the overall strain in the system; for instance, the $\mathrm{Zr}$ solute atom relaxes toward the NN vacancy by $0.45 \AA$ (see Fig. 2(b)). Such a large relaxation increases the energetic interaction between the solute and an accompanying vacancy. Thus, one can expect stronger solute $-\square$ binding energies with increase in the size of the solute/impurity atom.

Thus, solute- $\square$ binding energies seem to broadly correlate with the size of the transition metal solutes, as seen in Fig. 1. As cobalt is one of the smallest transition metals, all other substituted transition metals apply a positive strain (repulsive) on the NN Co atoms. Therefore, it is unsurprising that most solutes in fcc cobalt favor having a nearest-neighbor vacancy that reduces the strain in the lattice.

Interestingly, large solute atoms attract (i.e., bind weakly) with their $2 \mathrm{NN}$ host atoms. As seen in Fig. 3(c), large solute atoms such as Hf and Zr attract their six $2 \mathrm{NN}$ atoms making them relax inward toward the solute by about $0.03 \AA$. This inward relaxation of the $2 \mathrm{NN}$ host atoms becomes significantly larger when the solute atoms neighbor a vacancy. As seen in Fig. 2(b) the 
$\mathrm{Zr}-\mathrm{Co} 2 \mathrm{NN}$ distances in the (100) plane decrease from $3.46 \AA$ in $\mathrm{Co}_{107} \square_{1}$ to $3.14 \AA$ in $\mathrm{Co}_{106} \mathrm{Zr}_{1} \square_{1}$, approaching the $2.90 \AA \mathrm{Zr}-\mathrm{Co}$ bondlength in the ordered $\mathrm{Co}_{2} \mathrm{Zr}$ compound. This weak attraction with the $2 \mathrm{NN}$ host atoms leads to unfavorable $2 \mathrm{NN}$ solute- $\square$ binding energies for large solute atoms, as seen in Table 1. Therefore, a NN vacancy not only relaxes the strain associated with large solutes, but also enhances their bonding interaction with $2 \mathrm{NN}$ host atoms.

However, there are exceptions to this size-effect. For instance, Ti has a NN solute- $\square$ binding energy $(0.16 \mathrm{eV})$ much larger than that of $\mathrm{W}(0.06 \mathrm{eV})$, but its impurity volume is around $10 \%$ smaller. Though solute size is a large factor in controlling the solute- $\square$ binding energy, it is clearly not the only factor, and there is also an important electronic aspect influencing it. In the next section we consider $d$-band filling model which can serve as a complement to the above strain model.

\subsubsection{Effect of d-occupancy}

Fig. 4 shows that the NN solute- $\square$ binding energy follows a parabolic behavior as a function of the $d$-occupancy of the solute across the transition metal series with a minimum at the middle of the series. A similar parabolic trend is known for cohesive energy [42-44], vacancy formation energy [45], and solute- $\square$ interaction in other hosts $[18,37,46,47]$. In fact, the parabolic behavior of the solute- $\square$ binding energy can be explained as a band-filling effect using Friedel's model $[37,42,44]$, and its extension for $d$-band cohesion in pure transition metals [43]. As discussed in Ref. [44] the bonding energy $\left(U_{\text {bond }}^{\mathrm{AB}}\right)$ between transition metal atoms $A$ and $B$ is defined as:

$$
U_{\text {bond }}^{\mathrm{AB}}(\bar{N})=-\frac{1}{20} \bar{W}_{\mathrm{AB}} \bar{N}(10-\bar{N})
$$

where $\bar{N}=\frac{1}{2}\left(N_{\mathrm{d}}^{\mathrm{A}}+N_{\mathrm{d}}^{\mathrm{B}}\right)$ is the average number of $d$-electrons and $\bar{W}_{\mathrm{AB}}=$ ${ }_{230}\left[\frac{1}{2}\left(W_{\mathrm{A}}^{2}+W_{\mathrm{B}}^{2}\right)\right]^{1 / 2}$ is the average bandwidth of the $d$-band. Across a transition metal series, from $d^{1}$ to $d^{5}$ (early $d$ solutes), only the bonding states are filled and the bond strength gradually increases. Further, for the later transition metals, from $d^{6}$ to $d^{10}$, the anti-bonding states are also filled, thereby lowering the bond 
strength (here, between the solute and the host atoms). Therefore, energetics or comparison of bond strengths in transition metals (e.g., solute- $\square$ binding energy [37, 48]) always show a band-filling effect. In fact, this $d$-band filling effect is also visible in the effective displacement of host cobalt atoms during relaxation upon solute substitution nearest to a vacancy (see Figs. 3(b, d)). the middle of the series and decreases gradually towards either end (according to Eq. 8). This results in the NN host atoms undergoing small, negligible displacements away in the case of strongly-binding mid-transition metals, and much larger displacements for weakly-binding early- and late-transition metals 245 (see Fig. 3(b)). Further, the strong solute-NN host bonds in the case of midtransition metals (when compared to early-, late-transition metals) likely result in weaker NN-2NN host bonds, thus causing the $2 \mathrm{NN}$ host atoms to relax away from the solute atom (see the effective repulsive interactions between the solute and $2 \mathrm{NN}$ host atoms, especially for mid-4d, 5d solutes in Fig. 3(d)). For $3 d$ transition metal atoms, the solute- $\square$ binding energy parabola is somewhat "flattened" (Fig. 4), and a double well is seen (Fig. 3(b, d)) due to the loss of their magnetic moments [49], which is absent in the $4 d$ and $5 d$ transition metal series.

Further, this $d$-band filling effect is also observed in the calculated dilute 255 impurity formation energies $\left(E_{\mathrm{imp}}^{\mathrm{eq}}\right)$. As seen in Fig. 5, the impurity formation energy follows the $d$-filling across the transition metal series, with the exception of group 11 and magnetic $3 d$ transition metals. The flattening of $E_{\mathrm{imp}}^{\mathrm{eq}}$ in magnetic transition metals can be attributed to the loss of the atomic magnetic moment and the associated exchange energy compared to the free atom $[46,49]$.

\subsection{Solubility Enthalpy}

For all elemental solutes considered, we calculate their solubility enthalpy $\Delta H_{\text {sol }}$ in fcc cobalt as the difference between the formation enthalpy of the respective cobalt-rich ordered compound $\Delta H_{\mathrm{ord}}^{\mathrm{eq}}$ and the impurity formation en- 


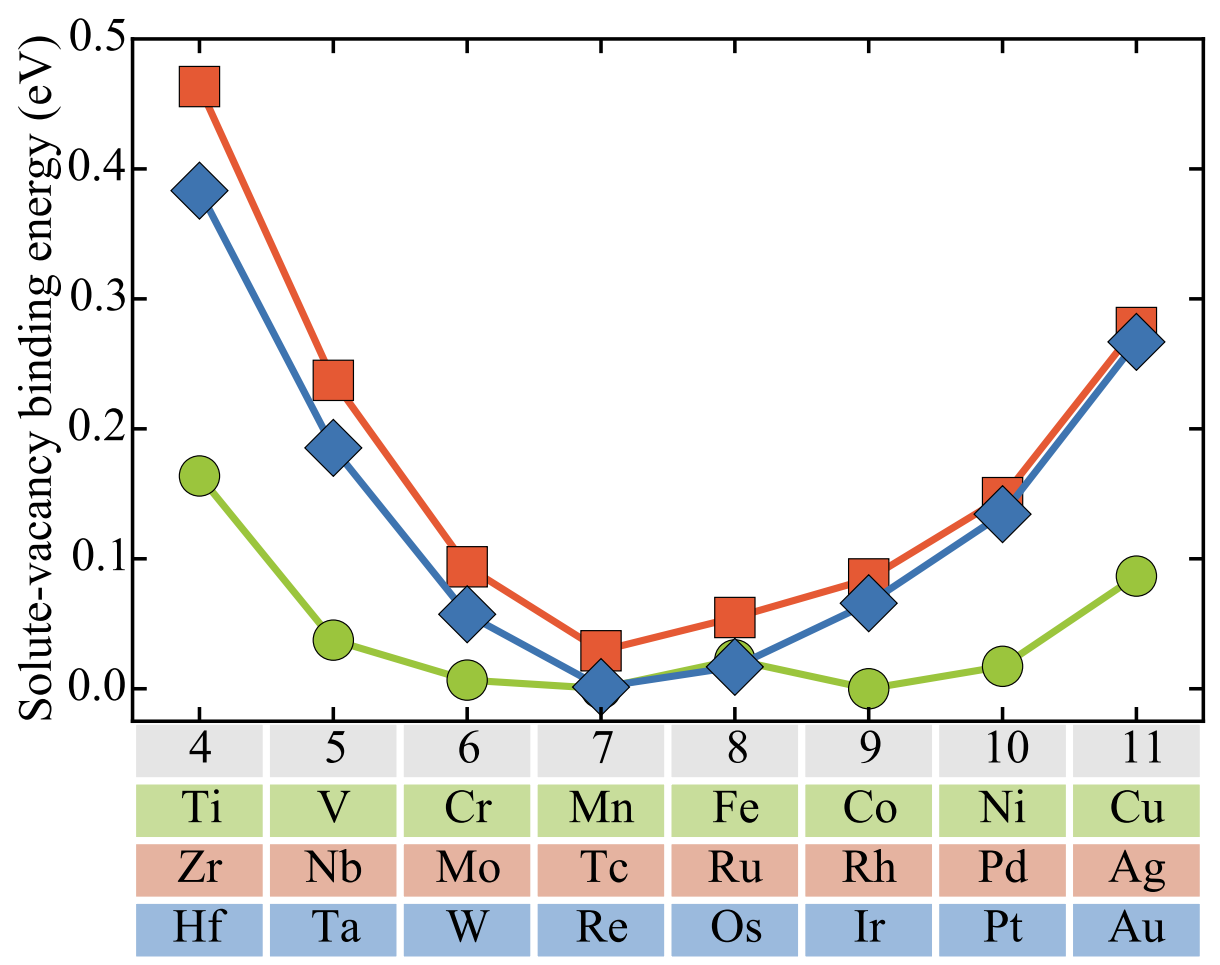

Figure 4: (Color online) The calculated solute-vacancy binding energies as a function of $d$ occupancy of the solute - the binding energies follow a parabolic trend across the periodic table, reaching a minimum at $d^{4-6}$ occupancies. 
Table 2: For each elemental solute $X$ considered in this paper, we list (a) its elemental equilibrium crystal structure, (b-e) chemical formula, structural prototype, space group, and formation energy of the Co-rich ordered phase reported in the Co- $X$ binary system, $(\mathrm{f}-\mathrm{g})$ the dilute impurity formation energy with respect to the $X$ in its equilibrium crystal structure $\left(E_{\mathrm{imp}}^{\mathrm{eq}}\right)$ and a hypothetical fcc structure $\left(E_{\mathrm{imp}}^{\mathrm{fcc}}\right)$ respectively, and (h) its solubility enthalpy $\Delta H_{\text {sol }}$ in fcc cobalt.

\begin{tabular}{|c|c|c|c|c|c|c|c|c|c|}
\hline & Solute & $\begin{array}{l}\text { Crystal } \\
\text { structure }\end{array}$ & $\begin{array}{l}\text { Chemical } \\
\text { formula }^{\dagger}\end{array}$ & $\begin{array}{l}\text { Structural } \\
\text { prototype }\end{array}$ & $\begin{array}{l}\text { Space } \\
\text { group }\end{array}$ & $\begin{array}{c}\Delta H_{\text {ord }}^{\mathrm{eq}} \\
(\mathrm{eV} / \text { atom })\end{array}$ & $\begin{array}{l}E_{\mathrm{imp}}^{\mathrm{eq}} \\
\quad(\mathrm{eV}\end{array}$ & $\begin{array}{l}E_{\mathrm{imp}}^{\mathrm{fcc}} \\
\text { solute }\end{array}$ & $\begin{array}{l}\Delta H_{\mathrm{sol}} \\
\mathrm{om})\end{array}$ \\
\hline & $\mathrm{Al}$ & fcc & CoAl [50] & $\mathrm{CsCl}$ & $\operatorname{Pm} \overline{3} \mathrm{~m}$ & -1.22 & -0.98 & -0.98 & -0.24 \\
\hline & $\mathrm{Si}$ & diamond & $\mathrm{Co}_{2} \mathrm{Si}[51]$ & $\mathrm{Co}_{2} \mathrm{Si}$ & Pnma & -1.39 & -1.06 & -1.60 & -0.33 \\
\hline \multirow{7}{*}{$\vec{m}$} & $\mathrm{Ti}$ & hcp & $\mathrm{Co}_{3} \mathrm{Ti}[52]$ & $\mathrm{AuCu}_{3}$ & $\operatorname{Pm} \overline{3} \mathrm{~m}$ & -1.09 & -0.70 & -0.75 & -0.39 \\
\hline & $\mathrm{V}$ & bcc & $\mathrm{Co}_{3} \mathrm{~V}[53]$ & $\mathrm{Al}_{3} \mathrm{Pu}$ & $\operatorname{Pm} \overline{3} \mathrm{~m}$ & -0.76 & -0.21 & -0.46 & -0.55 \\
\hline & $\mathrm{Cr}$ & bcc & - & - & - & 0.00 & 0.42 & 0.03 & -0.42 \\
\hline & $\mathrm{Mn}$ & $\alpha-\mathrm{Mn}$ & CoMn [54] & $\mathrm{CsCl}$ & $\operatorname{Pm} \overline{3} \mathrm{~m}$ & -0.02 & 0.30 & 0.22 & -0.32 \\
\hline & $\mathrm{Fe}$ & bcc & $\mathrm{CoFe}[55]$ & $\mathrm{CsCl}$ & $\operatorname{Pm} \overline{3} \mathrm{~m}$ & -0.13 & -0.01 & -0.16 & -0.12 \\
\hline & $\mathrm{Ni}$ & fcc & - & - & - & -0.04 & -0.04 & -0.04 & $\approx 0.0$ \\
\hline & $\mathrm{Cu}$ & fcc & - & - & - & 0.00 & 0.35 & 0.35 & -0.35 \\
\hline \multirow{8}{*}{ F } & $\mathrm{Zr}$ & hcp & $\mathrm{Co}_{2} \mathrm{Zr}[56]$ & $\mathrm{MgCu}_{2}$ & $\mathrm{Fd} \overline{3} \mathrm{~m}$ & -1.01 & 0.00 & -0.20 & -1.01 \\
\hline & $\mathrm{Nb}$ & bcc & $\mathrm{NbCo}_{3}[57]$ & $\mathrm{NbCo}_{3}$ & $\mathrm{P} 6_{3} / \mathrm{mm}$ & -0.48 & 0.12 & -0.21 & -0.60 \\
\hline & Mo & bcc & $\mathrm{Co}_{3} \mathrm{Mo}$ [58] & $\mathrm{Mg}_{3} \mathrm{Cd}$ & $\mathrm{P} 6_{3} / \mathrm{mm}$ & -0.30 & 0.70 & 0.28 & -1.00 \\
\hline & $\mathrm{Tc}$ & hcp & - & - & - & 0.00 & 0.80 & 0.73 & -0.80 \\
\hline & $\mathrm{Ru}$ & hcp & - & - & - & 0.00 & 0.67 & 0.56 & -0.67 \\
\hline & $\mathrm{Rh}$ & fcc & - & - & - & 0.00 & 0.18 & 0.18 & -0.18 \\
\hline & $\mathrm{Pd}$ & fcc & - & - & - & 0.00 & 0.31 & 0.31 & -0.31 \\
\hline & $\mathrm{Ag}$ & fcc & - & - & - & 0.00 & 1.42 & 1.42 & -1.42 \\
\hline \multirow{8}{*}{20} & $\mathrm{Hf}$ & hcp & $\mathrm{HfCo}_{2}[59]$ & $\mathrm{MgCu}_{2}$ & $\mathrm{Fd} \overline{3} \mathrm{~m}$ & -1.15 & -0.29 & -0.39 & -0.86 \\
\hline & $\mathrm{Ta}$ & bcc & $\mathrm{Co}_{3} \mathrm{Ta}[60]$ & $\mathrm{AuCu}_{3}$ & $\mathrm{Pm} \overline{3} \mathrm{~m}$ & -0.96 & -0.10 & -0.34 & -0.86 \\
\hline & $\mathrm{W}$ & bcc & $\mathrm{Co}_{3} \mathrm{~W}[58]$ & $\mathrm{Mg}_{3} \mathrm{Cd}$ & $\mathrm{P} 6_{3} / \mathrm{mm}$ & -0.40 & 0.61 & 0.12 & -1.01 \\
\hline & $\operatorname{Re}$ & hcp & - & - & - & 0.00 & 0.79 & 0.73 & -0.79 \\
\hline & Os & hcp & - & - & - & 0.00 & 0.81 & 0.67 & -0.81 \\
\hline & Ir & fcc & - & - & - & 0.00 & 0.13 & 0.13 & -0.13 \\
\hline & $\mathrm{Pt}$ & fcc & $\mathrm{CoPt}[61]$ & $\mathrm{AuCu}$ & $\mathrm{P} 4 / \mathrm{mmm}$ & -0.19 & -0.15 & -0.15 & -0.04 \\
\hline & $\mathrm{Au}$ & fcc & - & - & - & 0.00 & 0.77 & 0.77 & -0.77 \\
\hline
\end{tabular}

$\dagger$ for elements that form a solid solution with cobalt at all compositions or those that have no solubility in cobalt (indicated by - in the corresponding columns), the $\Delta H_{\mathrm{sol}}$ is reported simply as the negative of the impurity formation energy $\left(E_{\mathrm{imp}}^{\mathrm{eq}}\right)$. 

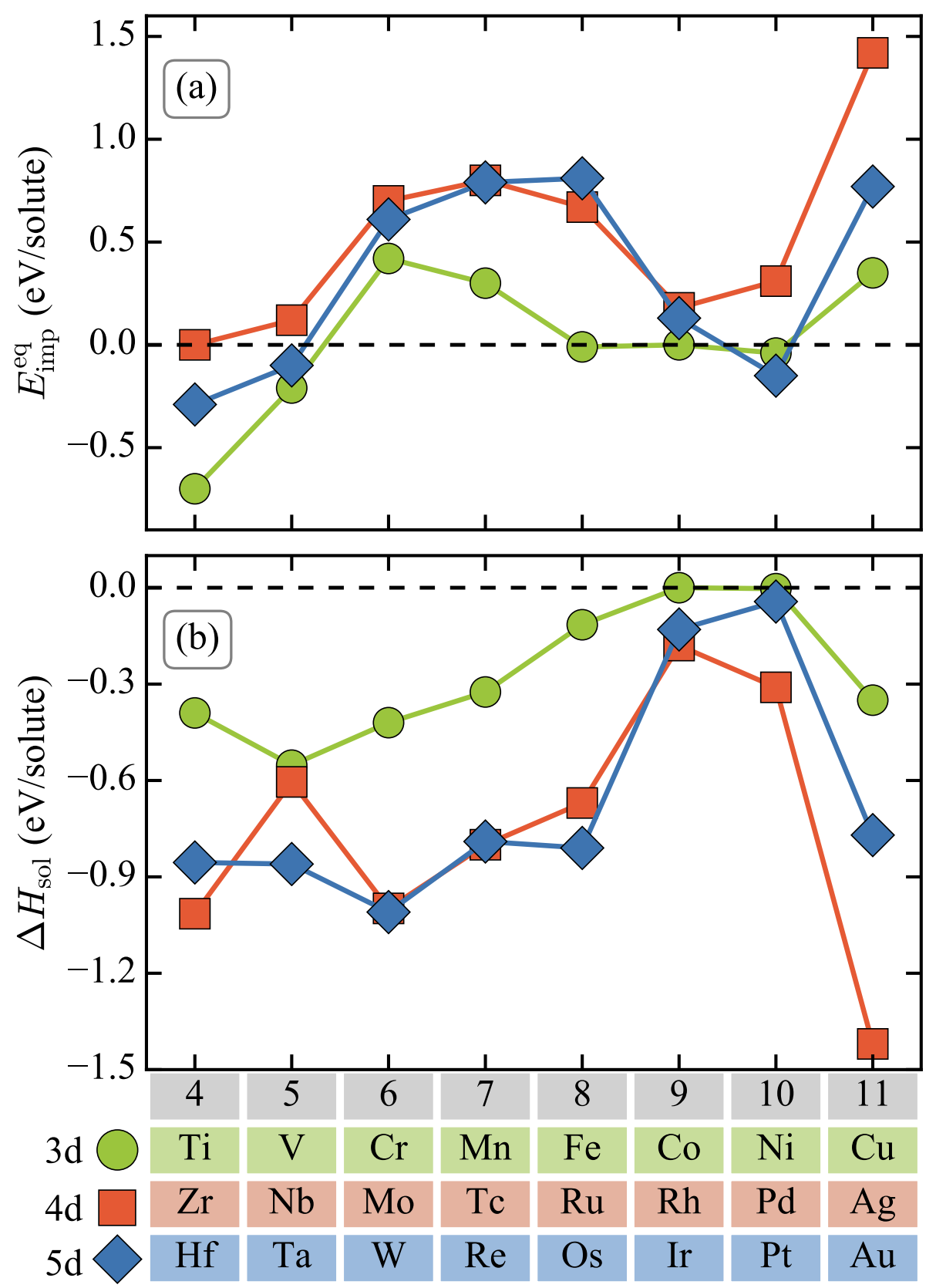

Figure 5: (Color online) The calculated (a) impurity formation energy, and (b) solubility enthalpy, as a function of $d$-occupancy, of all transition metal solutes considered in this work. 
ergy $E_{\text {imp }}^{\mathrm{eq}}$ (see Eq. 6). The results for $\Delta H_{\text {sol }}$ are shown in Table 2 and plotted in Fig. 5. The solubility enthalpy is directly related to the temperature dependence of solubility the respective solute, as described in Eq. 7-large negative values lead to low solubility or solubility only at high temperatures. Solubilities calculated using DFT have previously shown reasonable agreement with experimental data where available, e.g., in the case of $\mathrm{Al}[33,35,36]$.

As seen in Fig. 5, $4 d$ and $5 d$ transition metals, except those belonging to groups 9 and 10, have large negative $\Delta H_{\text {sol }}$ compared to $3 d$ transition metals. The large negative values for the majority of $4 d$ and $5 d$ solutes result in their low solubility in fcc cobalt, consistent with previous observations of their low solubility in almost all $3 d$ transition metal hosts [62]. For example, Ag with a very large negative value of $\Delta H_{\text {sol }}=-1.42 \mathrm{eV}$ has negligible solubility in fcc cobalt, in agreement with experimental phase diagrams [63], where little to no solubility is observed for temperatures below the melting point of cobalt. The calculated solubility data is roughly consistent with the size factor statement of Hume-Rothery rules [64]: the solubility is unlikely to be high when the atomic radius of the solute differs more than $\sim 15 \%$ from that of the host, here fcc cobalt. In terms of volume, this means that solutes with an impurity volume of roughly $\geq 6 \AA^{3}$ (see Fig. 1) are expected to have low solubility in fcc cobalt. Among the solutes considered in this work, only $\mathrm{Au}, \mathrm{Ag}$, Hf, and $\mathrm{Zr}$ fulfill this criterion and all of them are calculated to have very low solubility (seen in Fig. 5), consistent with experimental phase diagrams [65].

We found that $3 d$ transition metals generally have higher solubility than $4 d$ and $5 d$ ones. Among $3 d$ transition metals nickel has nearly zero $\Delta H_{\text {sol }}$ indicating nearly complete solubility (see Eq. 7), in agreement with experimental observations of a $\mathrm{Co}-\mathrm{Ni}$ solid solution for all compositions [66]. Among $4 d$ and $5 d$ transition metals those belonging to groups 9 and 10, i.e., $\mathrm{Pd}, \mathrm{Pt}, \mathrm{Rh}$ and $\mathrm{Ir}$, have exceptionally large solubility in fcc cobalt, even larger than many $3 d$ transition metals, according to our calculations. This is consistent with experimental phase diagrams of binary systems $\mathrm{Co} X(X=\mathrm{Pd}, \mathrm{Pt}, \mathrm{Rh}, \mathrm{Ir})[52,61,67]$, which show solid solutions at $1000 \mathrm{~K}$ for all compositions. While there is a 

diagrams data, for an accurate calculation of solubility, estimating the vibrational entropic contributions is likely important, even necessary [33, 35]. This will be the subject of future work.

\section{Conclusion}

We have presented here accurate density functional calculations of a host of thermodynamic properties for 25 different solutes in fcc cobalt, including solutevacancy binding energies, enthlapy of formation of ordered phases, impurity formation energies with respect to equilibrium and hypothetical fcc reference states, and solubility enthalpies.

We have studied the physical and chemical effects controlling solute- $\square$ interactions. We find that there is a strong correlation between solute- $\square$ binding energy and solute size, with larger solute atoms possessing a stronger binding with vacancies. This effect of solute size was understood in the terms of: (a) a strain-relief argument, in which a vacancy relieves the strain associated with large solute atoms, (b) secondary bonding interactions between the solute and the next-nearest neighbor atoms mediated by a vacancy.

Besides the effect of solute size, the solute- $\square$ binding energy follows a parabolic trend as a function of $d$-occupancy across the transition metal series, falling to a minimum at the middle of the series. This parabolic effect is explained in terms of a $d$-band filling effect, also known as Friedel's model. In the case of $3 d$ solutes in fcc cobalt, magnetic exchange effect increases the solute- $\square$ binding energies resulting in flattening of the parabolic trend compared to $4 d$ and $5 d$ transition metals. In general, $4 d$ and their counterpart $5 d$ transition metals have nearly similar solute-vacancy binding energies, much larger than those of $3 d$ transition metals.

The large number of thermodynamic properties calculated in this work not only helps in understanding phase stability of various phases in fcc cobalt but can also be used as input for calculating multicomponent phase diagrams using 
methods such as CALPHAD. Overall, our extensive first-principles investigation of thermodynamics in fcc cobalt will significantly accelerate the design and development of new cobalt-based superalloys.

\section{Acknowledgments}

SSN and CW were supported by The Center for Hierarchical Materials Design (CHiMaD), Dept. of Commerce, NIST award 70NANB14H012. VIH was supported by the National Science Foundation through grant DMR-1309957. The computational work was done using Quest High Performance Computing Cluster at Northwestern University, and resources of the National Energy Research Scientific Computing (NERSC) Center, a DOE Office of Science User Facility supported by the Office of Science of the U.S. Department of Energy under Contract No. DE-AC02-05CH11231.

[1] J. Sato, T. Omori, K. Oikawa, I. Ohnuma, R. Kainuma, K. Ishida, CobaltBase High-Temperature Alloys, Science 312 (5770) (2006) 90-91. doi: 10.1126/science. 1121738.

[2] M. Tsunekane, A. Suzuki, T. M. Pollock, Single-crystal solidification of new Co-Al-W-base alloys, Intermetallics 19 (5) (2011) 636-643. doi: 10.1016/j.intermet.2010.12.018.

[3] T. M. Pollock, J. Dibbern, M. Tsunekane, J. Zhu, a. Suzuki, New Co-based $\gamma-\gamma^{\prime}$ High-temperature alloys, Jom-J Min Met Mat S 62 (1) (2010) 58-63. doi:10.1007/s11837-010-0013-y.

[4] S. Miura, K. Ohkubo, T. Mohri, Mechanical Properties of Co-Based $\mathrm{L1}_{2}$ Intermetallic Compound $\mathrm{Co}_{3}(\mathrm{Al}, \mathrm{W})$, Mater. Trans. 48 (9) (2007) 24032408. doi:10.2320/matertrans. MAW200734.

[5] M. Ooshima, K. Tanaka, N. L. Okamoto, K. Kishida, H. Inui, Effects of quaternary alloying elements on the $\gamma^{\prime}$ solvus temperature of $\mathrm{Co}-\mathrm{Al}-\mathrm{W}$ based alloys with fcc/L1 $/ 2$ two-phase microstructures, J. Alloys Compd. 508 (1) (2010) 71-78. doi:10.1016/j.jallcom.2010.08.050. 
[6] A. Suzuki, H. Inui, T. M. Pollock, L1 $1_{2}-$ Strengthened Cobalt-Base Superalloys, Annu. Rev. Mater. Res. 45 (1) (2015) 345-368. doi:10.1146/ annurev-matsci-070214-021043.

355 [7] D. L. Klarstrom, Wrought cobalt- base superalloys, J. Mater. Eng. Perform. 2 (4) (1993) 523-530. doi:10.1007/BF02661736.

[8] H.-Y. Yan, V. Vorontsov, D. Dye, Alloying effects in polycrystalline $\gamma^{\prime}$ strengthened Co-Al-W base alloys, Intermetallics 48 (2014) 44-53. doi: $10.1016 / j$. intermet.2013.10.022.

[9] J. Koßmann, T. Hammerschmidt, S. Maisel, S. Müller, R. Drautz, Solubility and ordering of $\mathrm{Ti}$, Ta, $\mathrm{Mo}$ and $\mathrm{W}$ on the $\mathrm{Al}$ sublattice in $\mathrm{L}_{2}-\mathrm{Co}_{3} \mathrm{Al}$, Intermetallics 64 (2015) 44-50. doi:10.1016/j.intermet.2015.04.009.

[10] A. Bauer, S. Neumeier, F. Pyczak, R. Singer, M. Göken, Creep properties of different $\gamma^{\prime}$-strengthened Co-base superalloys, Mater. Sci. Eng. A 550 (2012) 333-341. doi:10.1016/j.msea.2012.04.083.

[11] S. Kobayashi, Y. Tsukamoto, T. Takasugi, The effects of alloying elements (Ta, Hf) on the thermodynamic stability of $\gamma^{\prime}-\mathrm{Co}_{3}(\mathrm{Al}, \mathrm{W})$ phase, Intermetallics 31 (2012) 94-98. doi:10.1016/j.intermet.2012.06.006.

[12] A. Suzuki, High-temperature strength and deformation of $\gamma / \gamma^{\prime}$ two-phase Co-Al-W-base alloys, Acta Mater. 56 (6) (2008) 1288-1297. doi:10.1016/ j.actamat. 2007.11 .014 .

[13] D. Simonovic, M. H. F. Sluiter, Impurity diffusion activation energies in Al from first principles, Phys. Rev. B 79 (5) (2009) 054304. doi:10.1103/ PhysRevB.79.054304.

[14] C. Wolverton, Solute-vacancy binding in aluminum, Acta Mater. 55 (17) (2007) 5867-5872. doi:10.1016/j.actamat.2007.06.039.

[15] J. E. Saal, C. Wolverton, Solute-vacancy binding of the rare earths in magnesium from first principles, Acta Mater. 60 (13-14) (2012) 5151-5159. doi:10.1016/j.actamat.2012.06.016. 
[16] B. RW, H. PS., In: Diffusion metals park., OH: Am Soc Metals, 1973, p 83.

[17] D. Shin, C. Wolverton, First-principles study of solute vacancy binding in magnesium, Acta Mater. 58 (2010) 531-540. doi:10.1016/j.actamat. 2009.09 .031$.

[18] Y. Wang, H. Gao, Y. Han, Y. Dai, F. Bian, J. Wang, B. Sun, Firstprinciples study of solute-vacancy binding in $\mathrm{Cu}$, J. Alloys Compd. 608 (2014) 334-337. doi:10.1016/j.jallcom.2014.04.053.

[19] N. Saunders, A. P. Miodownik, CALPHAD (calculation of phase diagrams): a comprehensive guide, Vol. 1, Elsevier, 1998.

[20] P. Spencer, Computer simulations from thermodynamic data: Materials protection and development, MRS Bulletin 24 (1999) 18-21. doi:10.1557/ S088376940005212X.

[21] H. B. L. Kaufman, Computer Calculations of Phase Diagrams, Academic Press, New York, 1970.

[22] A. Costa e Silva, F. Rizzo, Summary report of XL CALPHADRio de Janeiro, Brasil, 2011, Calphad 39 (2012) 111-169. doi:10.1016/j. calphad.2012.07.003.

[23] C. Wolverton, X.-Y. Yan, R. Vijayaraghavan, V. Ozoliņš, Incorporating first-principles energetics in computational thermodynamics approaches, Acta Mater. 50 (9) (2002) 2187-2197. doi:10.1016/S1359-6454(01) 00430-X.

[24] Y. Zhong, C. Wolverton, Y. A. Chang, Z. K. Liu, A combined CALPHAD/first-principles remodeling of the thermodynamics of Al-Sr: Unsuspected ground state energies by "rounding up the (un)usual suspects", Acta Mater. 52 (9) (2004) 2739-2754. doi:10.1016/j.actamat. 2004.02 .022 . 
[25] P. E. Blöchl, Projector augmented-wave method, Phys. Rev. B 50 (1994) 17953-17979. doi:10.1103/PhysRevB.50.17953.

[26] G. Kresse, D. Joubert, From ultrasoft pseudopotentials to the projector augmented-wave method, Phys. Rev. B 59 (1999) 1758-1775. doi:10. 1103/PhysRevB.59.1758.

[27] G. Kresse, J. Furthmüller, Efficient iterative schemes for ab-initio totalenergy calculations using a plane-wave basis set, Phys. Rev. B 54 (1996) 11169-11186. doi:10.1103/PhysRevB.54.11169.

[28] G. Kresse, J. Furthmüller, Efficiency of ab-initio total energy calculations for metals and semiconductors using a plane-wave basis set, Comput. Mater. Sci. 6 (1) (1996) 15-50. doi:10.1016/0927-0256(96)00008-0.

[29] J. P. Perdew, K. Burke, M. Ernzerhof, Generalized gradient approximation made simple, Phys. Rev. Lett. 78 (1997) 1396-1399. doi:10.1103/ PhysRevLett.78.1396.

[30] M. Methfessel, A. T. Paxton, High-precision sampling for Brillouin-zone integration in metals, Phys. Rev. B 40 (1989) 3616-3621. doi:10.1103/ PhysRevB . 40.3616.

[31] J. D. Pack, H. J. Monkhorst, "Special points for Brillouin-zone integrations"-a reply, Phys. Rev. B 16 (1977) 1748-1749. doi:10.1103/ PhysRevB . 16.1748.

[32] P. E. Blöchl, O. Jepsen, O. K. Andersen, Improved tetrahedron method for Brillouin-zone integrations, Phys. Rev. B 49 (1994) 16223-16233. doi: 10.1103/PhysRevB.49.16223.

430

[33] C. Ravi, C. Wolverton, V. Ozoliņš, Predicting metastable phase boundaries in $\mathrm{AlCu}$ alloys from first-principles calculations of free energies: The role of atomic vibrations, Europhys. Lett. 73 (5) (2006) 719-725. doi:10.1209/ epl/i2005-10462-x. 
[34] Z. Mao, D. N. Seidman, C. Wolverton, First-principles phase stability, magnetic properties and solubility in aluminumrare-earth (AlRE) alloys and compounds , Acta Materialia 59 (9) (2011) 3659-3666. doi:10.1016/j. actamat.2011.02.040.

[35] V. Ozolinšs, M. Asta, Large Vibrational Effects upon Calculated Phase Boundaries in Al-Sc, Phys. Rev. Lett. 86 (2001) 448-451. doi:10.1103/ PhysRevLett. 86.448 .

[36] C. Wolverton, V. Ozolinšs, First-principles aluminum database: Energetics of binary Al alloys and compounds, Phys. Rev. B 73 (14) (2006) 144104. doi:10.1103/PhysRevB.73.144104.

[37] U. Klemradt, B. Drittler, T. Hoshino, R. Zeller, P. H. Dederichs, N. Stefanou, Vacancy-solute interactions in $\mathrm{Cu}, \mathrm{Ni}, \mathrm{Ag}$, and $\mathrm{Pd}$, Physical Review B 43 (12) (1991) 9487-9497. doi:10.1103/PhysRevB.43.9487.

[38] S. Neumeier, H. Rehman, J. Neuner, C. Zenk, S. Michel, S. Schuwalow, J. Rogal, R. Drautz, M. Göken, Diffusion of solutes in fcc Cobalt investigated by diffusion couples and first principles kinetic Monte Carlo, Acta Mater. 106 (2016) 304-312. doi:10.1016/j.actamat.2016.01.028.

[39] a. K. Shukla, C. Biswas, R. S. Dhaka, S. C. Das, P. Krüger, S. R. Barman, Influence of $s p-d$ hybridization on the electronic structure of Al-Mn alloys, Phys. Rev. B 77 (19) (2008) 195103. doi:10.1103/PhysRevB.77.195103.

[40] G. Frenking, N. Fröhlich, The Nature of the Bonding in TransitionMetal Compounds, Chem. Rev. 100 (2) (2000) 717-774. doi:10.1021/ cr9804011.

[41] J. S. Márquez, M. F. Núñez, On the determination of bond lengths by ab initio methods: estimation of errors and some improvements, Journal of Molecular Structure: THEOCHEM 624 (1) (2003) 239-249. doi:10.1016/ 
[42] J. M. Ziman (Ed.), The Physics of Metals, Cambridge: Cambridge University Press, 1969.

[43] A. P. Sutton, Electronic Structure of Materials, Oxford University Press, 2004.

[44] D. Pettifor, Bonding and Structure of Molecules and Solids, Oxford science publications, Clarendon Press, 1995.

[45] P. A. Korzhavyi, I. A. Abrikosov, B. Johansson, A. V. Ruban, H. L. Skriver, First-principles calculations of the vacancy formation energy in transition and noble metals, Physical Review B 59 (18) (1999) 11693-11703. doi: 10.1103/PhysRevB.59.11693.

[46] D. J. Hepburn, E. MacLeod, G. J. Ackland, Transition metal solute interactions with point defects in fcc iron from first principles, Physical Review B 92 (1) (2015) 014110. doi:10.1103/PhysRevB.92.014110.

[47] O. Gorbatov, P. Korzhavyi, A. Ruban, B. Johansson, Y. Gornostyrev, Vacancysolute interactions in ferromagnetic and paramagnetic bcc iron: $\mathrm{Ab}$ initio calculations, Journal of Nuclear Materials 419 (1-3) (2011) 248-255. doi:10.1016/j.jnucmat.2011.09.002.

[48] N. Stefanou, N. Papanikolaou, P. H. Dederichs, Solute-vacancy interactions in $\mathrm{Cu}$ and Ag, Journal of Physics: Condensed Matter 3 (45) (1991) 87938801. doi:10.1088/0953-8984/3/45/004.

[49] M. S. S. Brooks, B. Johansson, Exchange integral matrices and cohesive energies of transition metal atoms, Journal of Physics F: Metal Physics 13 (10) (1983) L197-L202. doi:10.1088/0305-4608/13/10/003.

[50] H. Ohtani, M. Yamano, M. Hasebe, Thermodynamic analysis of the CoAlC 485 and NiAlC systems by incorporating ab initio energetic calculations into the CALPHAD approach, Calphad 28 (2) (2004) 177-190. doi:10.1016/ j.calphad.2004.08.003. 
[51] L. Zhang, Y. Du, H. Xu, Z. Pan, Experimental investigation and thermodynamic description of the CoSi system, Calphad 30 (4) (2006) 470-481. doi:10.1016/j.calphad.2006.06.001.

[52] T. B. Massalski, H. Okamoto, P. Subramanian, L. Kacprzak, W. W. Scott, Binary alloy phase diagrams, Vol. 1, American Society for Metals Metals Park, OH, 1986.

[53] J. Bratberg, B. Sundman, A thermodynamic assessment of the CoV system, J. Phase Equilibria 24 (6) (2003) 495-503. doi:10.1361/ 105497103772084534.

[54] K. Ishida, T. Nishizawa, The Co-Mn (Cobalt-Manganese) system, Bull. Alloy Phase Diagrams 11 (2) (1990) 125-137. doi:10.1007/BF02841695.

[55] Y. I. Ustinovshikov, B. E. Pushkarev, I. V. Sapegina, Phase separation and ordering in the Fe-Co system, Inorg. Mater. 42 (4) (2006) 354-359. doi:10.1134/S0020168506040042.

[56] A. Durga, K. Hari Kumar, Thermodynamic optimization of the CoZr system, Calphad 34 (2) (2010) 200-205. doi:10.1016/j.calphad.2010.02. 006.

[57] H. Okamoto, Co-Nb (Cobalt-Niobium), J. Phase Equilibria Diffus. 31 (1) (2010) 94-95. doi:10.1007/s11669-009-9632-9.

[58] C. Wang, J. Wang, S. Guo, X. Liu, I. Ohnuma, R. Kainuma, K. Ishida, Experimental investigation and thermodynamic calculation of the phase equilibria in the CoMoW system, Intermetallics 17 (8) (2009) 642-650. doi:10.1016/j.intermet.2009.02.004.

[59] J. Bratberg, B. Jansson, Thermodynamic evaluation of the CCoWHfZr system for cemented carbides applications, J. Phase Equilibria Diffus. 27 (3) (2006) 213-219. doi:10.1361/154770306X109746. 
[60] K. C. H. Kumar, T. Van Rompaey, P. Wollants, Thermodynamic calculation of the phase diagram of the CoNbTa system, Zeitschrift für Met. 93 (11) (2002) 1146-1153. doi:10.3139/146.021146.

[61] H. Okamoto, Co-pt (cobalt-platinum), Journal of Phase Equilibria 22 (5) (2001) 591-591. doi:10.1361/105497101770332848.

[62] H. P. J. Wijn, Alloys and compounds of $3 \mathrm{~d}$ elements and $4 \mathrm{~d}$ or $5 \mathrm{~d}$ elements, Springer Berlin Heidelberg, 1991, pp. 68-94. doi:10.1007/ 978-3-642-58218-9_4.

[63] W. Zhu, H. Liu, J. Wang, H. Dong, Z. Jin, Thermodynamic assessment of the SnAgCo system and solidification simulation of the ternary alloy , Journal of Alloys and Compounds 481 (12) (2009) 503 - 508. doi:10. $1016 / j \cdot j a l l c o m .2009 .03 .025$.

[64] W. Hume-Rothery, H. M. Powell, On the theory of super-lattice structures in alloys, Z. Kristallogr. 91 (1) (1935) 23-47.

[65] H. Okamoto, K. Cenzual (Eds.), ASM Alloy Phase Diagrams Database, ASM International, Materials Park, OH, 2006.

530 [66] F. Guillermet, Assessment of the thermodynamic properties of the Ni-Co system, Zeitschrift für Metallkunde 78 (1987) 639-647.

[67] G. Ghosh, C. Kantner, G. B. Olson, Thermodynamic modeling of the Pd$\mathrm{X}(\mathrm{X}=\mathrm{Ag}, \mathrm{Co}, \mathrm{Fe}, \mathrm{Ni})$ systems, Journal of Phase Equilibria 20 (3) (1999) 295-308. doi:10.1361/105497199770335811. 


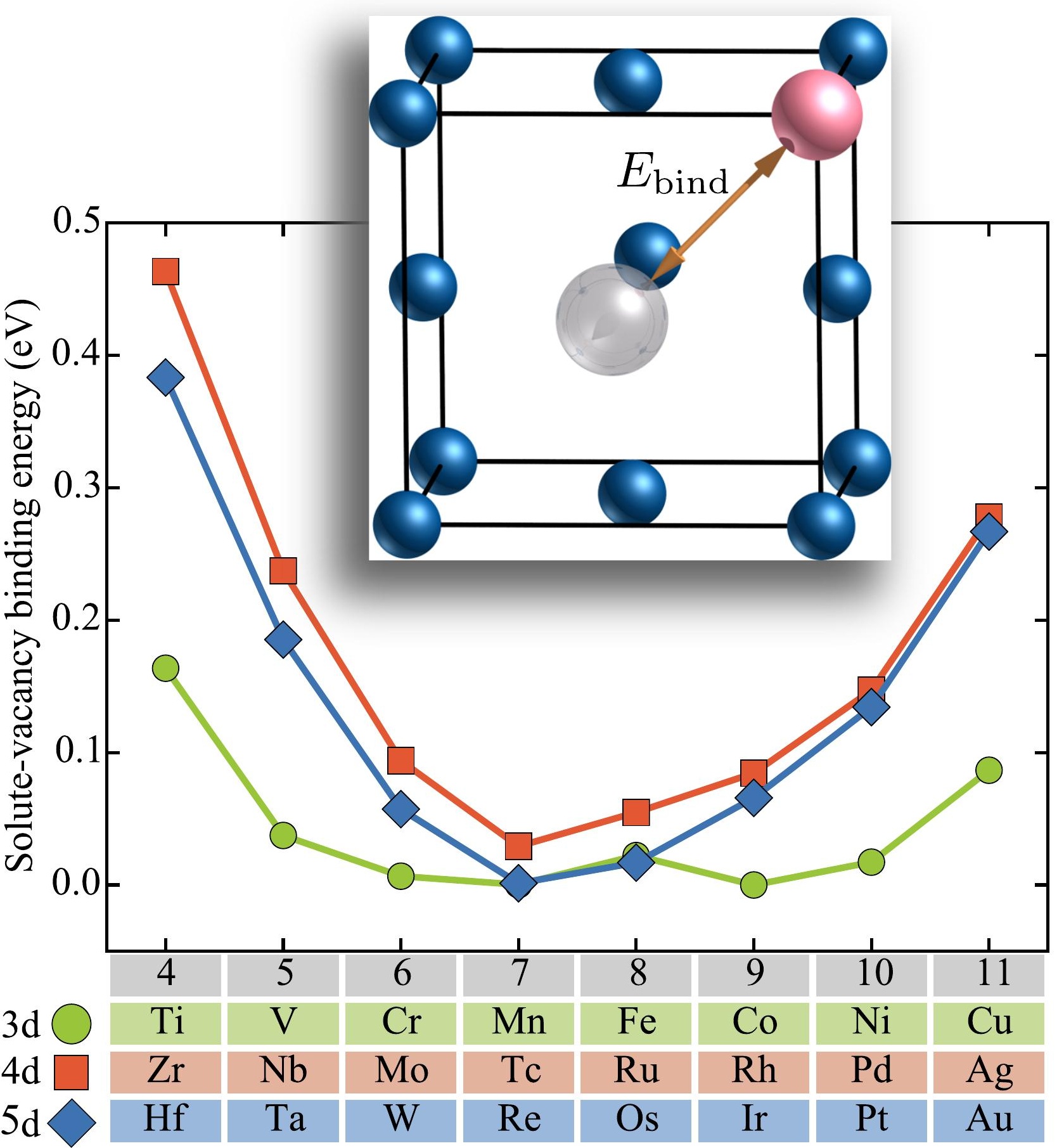

